\title{
Fiber-Optic Measurement of Temperature Profiles
}

\author{
Dirk Samiec, Jörg Schwarz \\ Polytec GmbH, Waldbronn, Germany \\ E-Mail: j.schwarz@polytec.de
}

\begin{abstract}
Temperature measurements in industrial or R\&D applications are conventionally based on thermocouples. But the single point method fails, if continuous temperature profiles are required at a specific date. Glass fibers are light weight, minimal invasive, can easily be integrated and used to measure in liquids, strong electro-magnetic or RF fields. Installation is easy and time saving. Temperature profiles with spatial resolution in the order of millimeters allow a much deeper understanding of heat exchange or flow processes. They help to allocate local hot-spots, feed existing FEM models and improve the degree of efficiency and safety wherever temperature counts.
\end{abstract}

Key Words: distributed sensing, fiber-optic, OFDR, temperature profile, Rayleigh sensing

\section{High Definition Rayleigh Sensing}

The heart of high definition (HD) sensing technology is a tunable laser source and a MachZehnder interferometric optical set-up as a combination of an active monitoring unit and a passive sensor fiber. Light guided through the fiber is back-scattered due to slight local variations of the refractive index of the material, waveguide geometry distortions or local defects. The scattered signals are superposed with the internal reference path of the interferometer. The sum signal now contains all information needed. After discrete Fourier transformation (FFT) each frequency value corresponds to a certain fiber position. That is why the method is called optical frequency domain reflectometry (OFDR). Once a commercially available glass fiber is scanned using OFDR a Rayleigh back-scatter signal as shown in Figure 1 is obtained.

There are slight variations of intensity along the fiber that remain very stable and that are highly reproducible as long as external conditions remain constant. Therefore, the amplitude distribution represents the "fingerprint" of a fiber or fiber segment, which is unique and can be used to evaluate temperature or strain data.

Compared to the so called OTDR technology the spatial resolution $d_{\min }$ of the collected raw data does not depend on the time of flight the light takes through the fiber. Respective interrogators reach approximately $1 \mathrm{~m}$ resolution. For the
OFDR method and set-up described above the spatial resolution is determined by the spectral width of the tunable laser source (e.g. $\Delta \lambda=80 \mathrm{~nm}$ $\rightarrow \mathrm{d}_{\min }=10 \mu \mathrm{m}$ ). For data evaluation an adequate software window (of e.g. $2.5 \mathrm{~mm}$ width) is selected. After FFT any frequency shift within such a window will be converted into a corresponding temperature value (see Figure 2). Commercially available OFDR systems allow sensing with millimeter spatial resolution up to $70 \mathrm{~m}$ of fiber length.

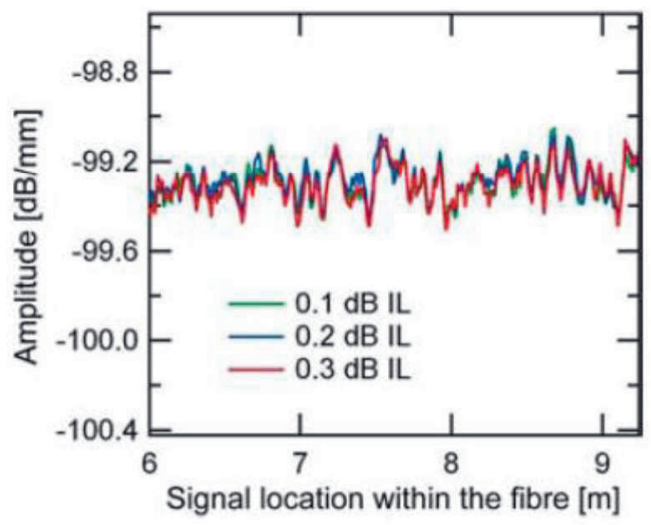

Fig. 1: Rayleigh back-scatter signal of fiber segment. For constant external conditions the profile remains the same for consecutive scans ("fingerprint").

Alternative back-scatter methods are Raman or Brillouin scattering. These technologies are used for long-distance applications in the $\mathrm{km}$ range having $1 \mathrm{~m}$ and down to some tens of centimeter spatial resolution. 


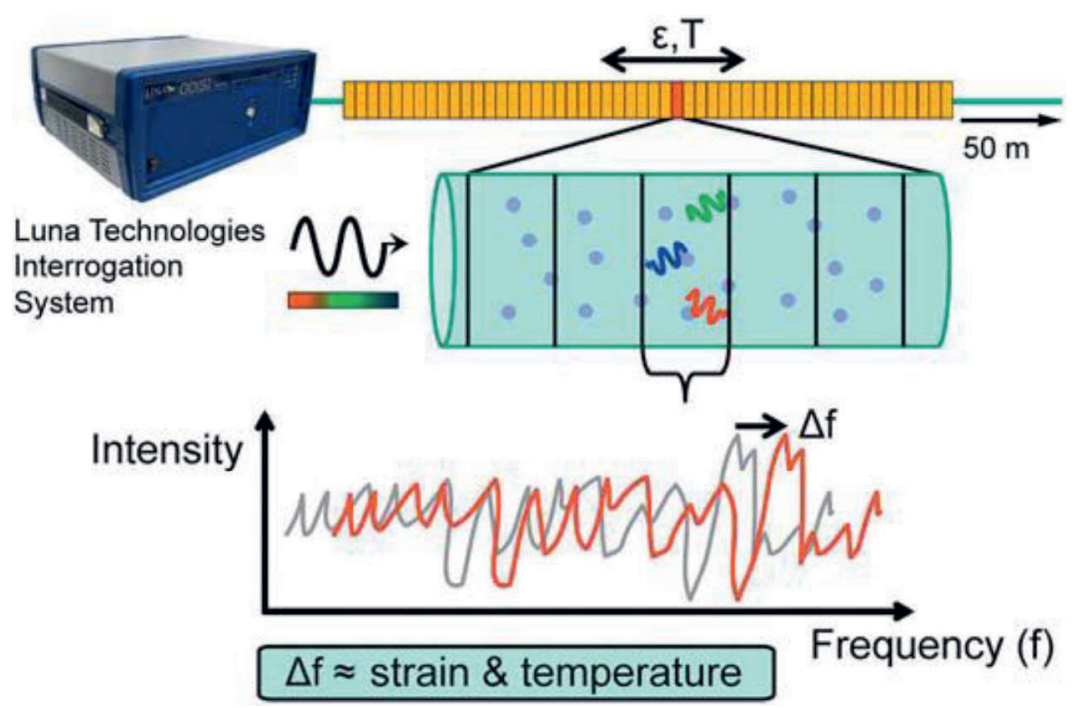

Fig. 2: The fiber is divided into small software windows. After Fourier transformation the Rayleigh signal in each window corresponds to a certain frequency. Any local frequency shift then is converted into temperature values.

\section{Process Engineering Applications}

Typical Rayleigh sensing applications in chemical and thermal engineering as well as in R\&D fields in institutes and universities are straight tube reactors like condensers, evaporators, distillers, ovens or extruders. Typical values of length or height are several meters. Detailed spatially and temporary resolved data for thermal processes are often not present. Certain operating modes may cause local mechanical damages due to a thermal inhomogeneity or hot spot. This is where distributed HD sensing is helpful and provides data for a better process understanding. It's a powerful tool to improve the degree of efficiency or safety, it may reduce service intervals or feed existing FEM models.

To improve the tube reactor geometry, flow conditions and the heat transfer, the TU Braunschweig in cooperation with CalGavin Ltd. uses a Rayleigh system at a straight-tube condenser of $2 \mathrm{~m}$ height (see Figure 3), where spatial condensation of Hexanol strongly depends on e.g. cooling agent temperature. Another goal was to study the condensation behavior for different reactor geometries, pressure and Hexanol volume flow.

Temporal and spatial resolution of the measurements had been $1 \mathrm{~Hz}$ and $1 \mathrm{~mm}$. The local shift of Hexanol condensation point influenced by the temperature of cooling agent is shown in Figure 4. The condensation point allocates the end and beginning of two phases that exist in the reactor. For $40^{\circ} \mathrm{C}$ condensation is almost complete at $0.8 \mathrm{~m}$. This location is moving downwards when cooling water temperature is increased to $60^{\circ} \mathrm{C}$. Moreover, for $80^{\circ} \mathrm{C}$ of cooling agent the evaporated Hexanol at given volume flow and pressure cannot be completely condensed anymore. This information gives significant input to fine-tune the condenser operating conditions for improved mass transfer per unit time.

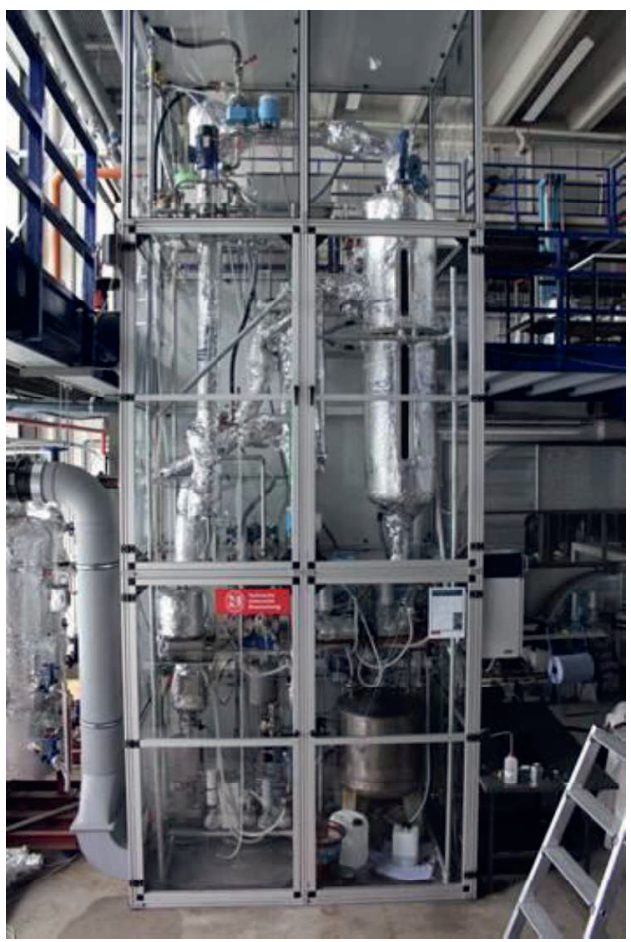

Fig. 3: Straight-tube condenser (TU Braunschweig) 


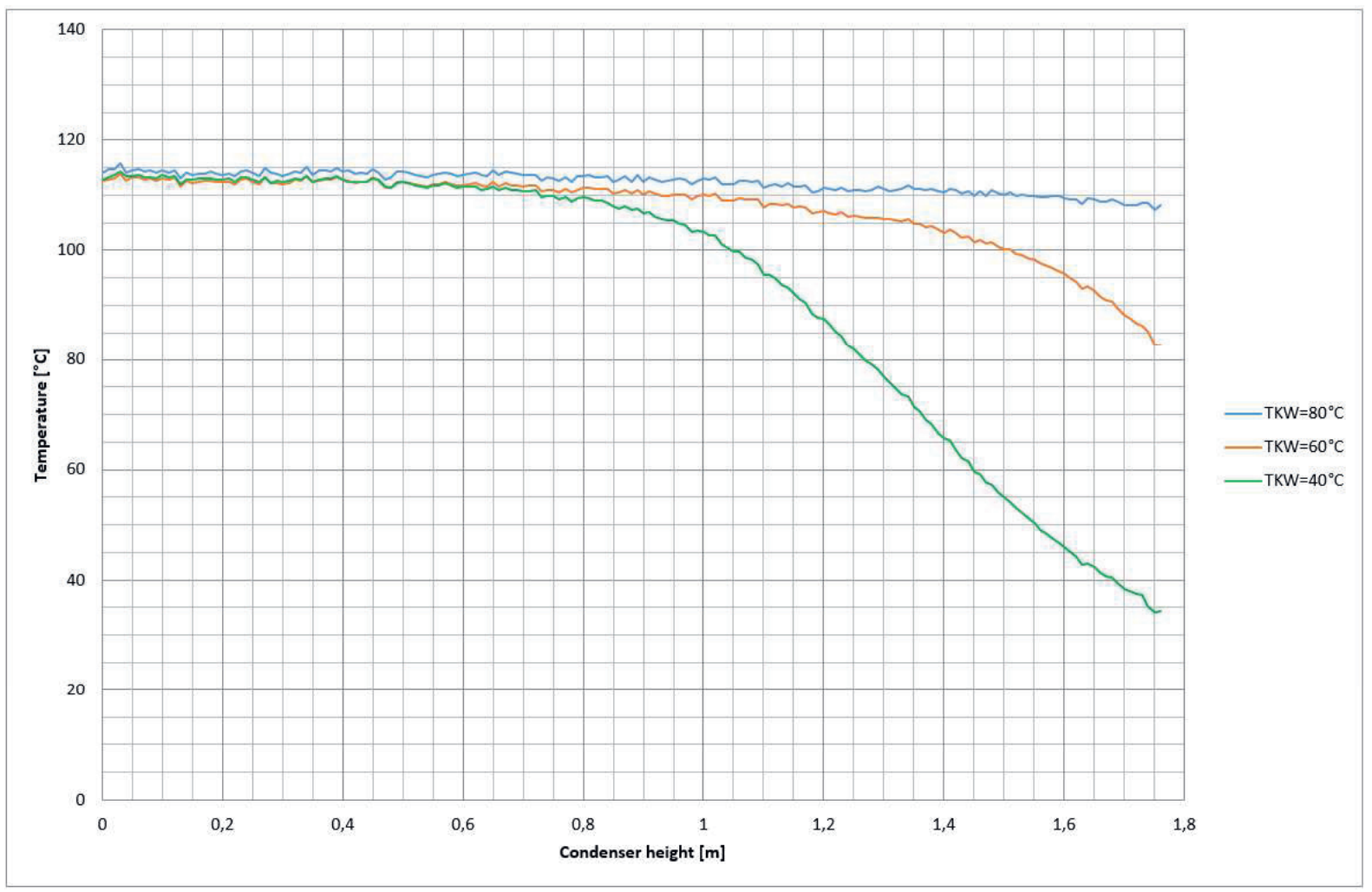

Fig.4: Temperature profiles of Hexanol in condenser for different cooling agent temperatures

The company SMS group $\mathrm{GmbH}$ used a Rayleigh system for HD temperature sensing to learn about highly distributed temperature profiles in their industrial large scale floatation ovens and segments thereof, each being several meters in length (see Figure 5). The preset temperature, gas flow and field is generated and maintained by a computer controlled complex structure of air nozzles. Their design and arrangement are customized.

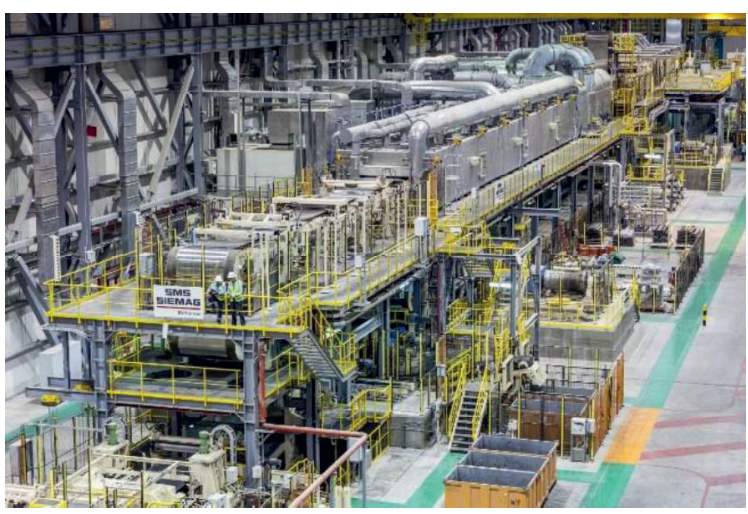

Fig. 5: SMS group $\mathrm{GmbH}$ floatation oven for generation of highly homogeneous large scale temperature fields
The thermal treatment of Aluminum strips for aircraft or automotive industry requires an exceedingly constant air flow and temperature distribution especially in the drying segment for thin films within the production line. For Rayleigh sensing the capillary guided fiber was placed into a pre-installed tube over approximately $10 \mathrm{~m}$ length, thus, addressing 4000 data points within minutes of installation time.

Figure 6 shows the temperature distribution measured with a LUNA ODiSI-B with $1 \mathrm{~Hz}$ and $2.5 \mathrm{~mm}$ resolution. By means of $\mathrm{HD}$ sensing technology it could be proven that for various temperature level air nozzles and temperature control are within customers' strict tolerances.

The different temperature level are also used for calibrating the sensor fiber based on reference data obtained with thermo-couples. 


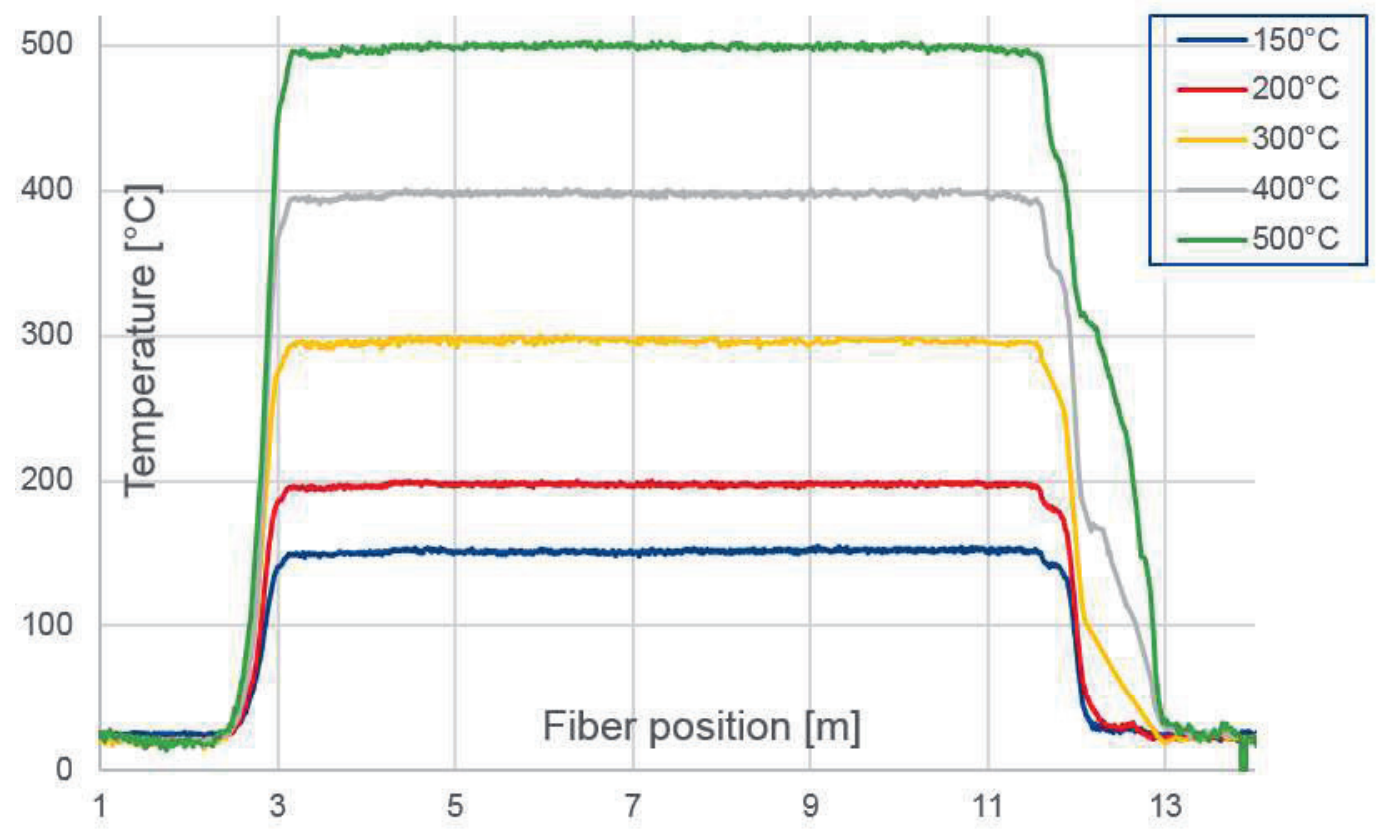

Fig. 6: Temperature distribution for different operating modes in a floatation oven segment; Within the treatment section between 5 and about $10.5 \mathrm{~m}$ the temperature field remains constant within $1.6 \mathrm{~K}$ around $500^{\circ} \mathrm{C}$

The PANDA facility at Swiss PSI (Paul-ScherrerInstitute) is investigating cooling systems for passive radioactive decay processes as in nuclear power plants. Especially the temperature distribution for simulated accidents in high pressure safety tanks was of interest. Panda is modular and flexible, which allows flexible modifications for either pressure tank, water basin or condenser operation and investigations therein. Within a vessel of total volume $460 \mathrm{~m}^{3}$ and height $25 \mathrm{~m}$ injected gas and steam of maximum temperature $200^{\circ} \mathrm{C}$ and 10 bar pressure realistic operating conditions are realized (see Figure 7 ).

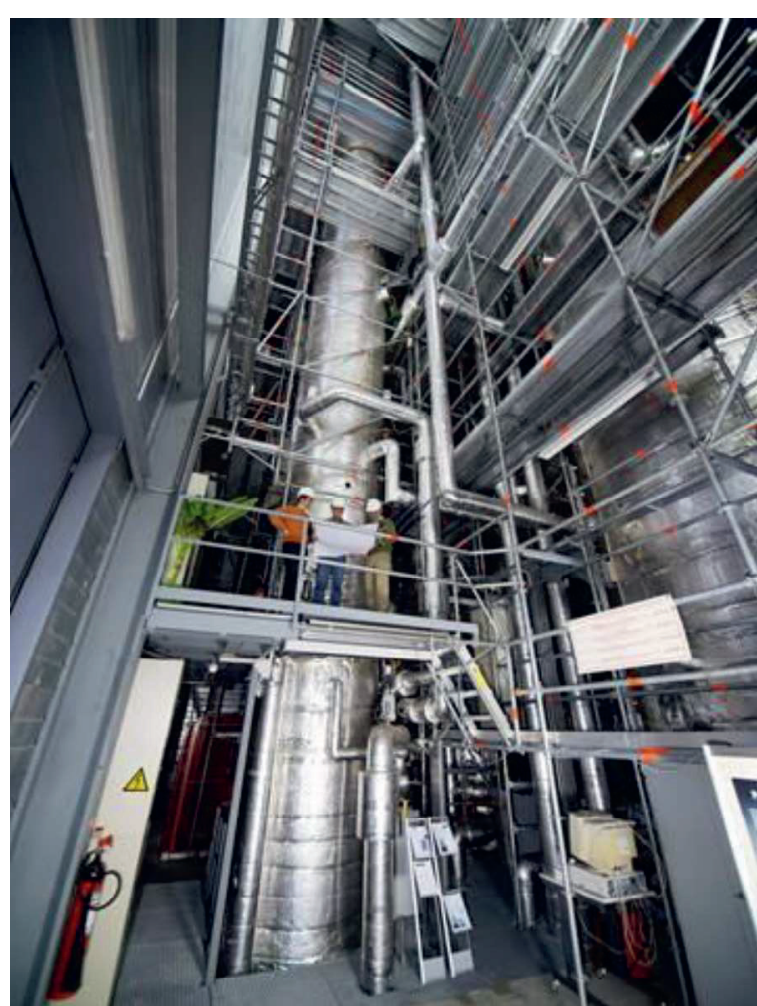

Fig. 7: Reactor pressure tank of PANDA at Swiss PSI (source: Paul-Scherrer-Institute) 
For HD temperature sensing a sensor fiber guided in a stainless steel capillary was horizontally mounted in the lower section of such vessel crossing a sparger ring cap for gas injection. Then hot air in combination with water steam are injected and volume flow was varied between 15 and 50g/s. Figure 8 gives an example that shows the temperature distribution in the pressure tank with/without the sparger ring. The modified flow conditions now cause a completely different temperature level and distribution in radial direction. The formerly sharp

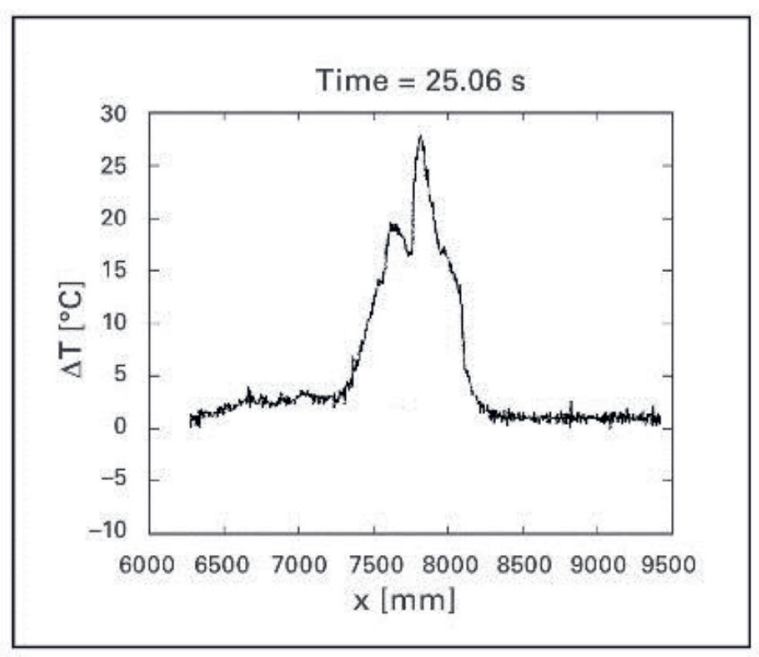

single maximum is now split into two of different shape and position that strongly affects cooling efficiency.

Earlier measurements had been done under similar operating conditions based on approximately 300 thermo-couples. The latest fiber optical HD sensing efforts deliver data with much higher point density on significantly shorter time scale and, maybe most important, prior installation time was considerably reduced and still is for any future modifications of set-up.

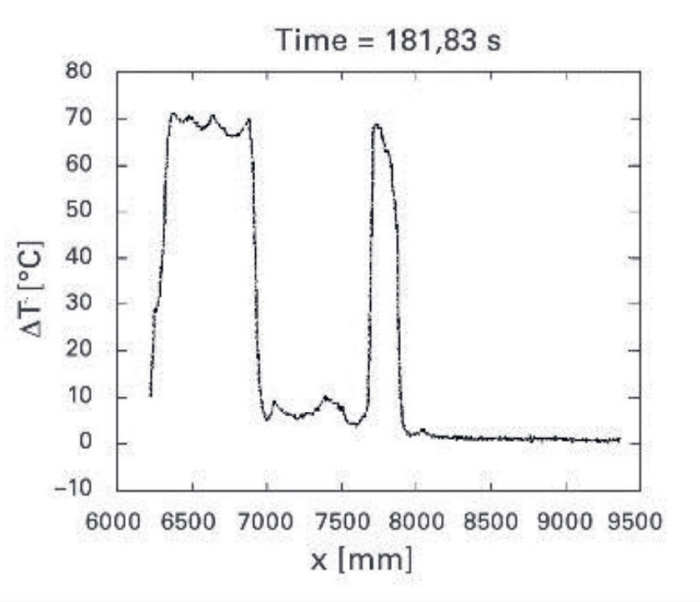

Fig. 8: Radial temperature distribution in PANDA high pressure tank with (left) and without (right) sparger ring

\section{Literature}

[1] J.M. Henault et al., "Qualification of a truly distributed fiber optic technique for strain and temperature measurements in concrete structures", EPJ Web Conferences 12, 03004 (2011)

[2] D. Samiec, Polytec GmbH, "Verteilte faseroptische Temperatur- und Dehnungsmessung mit sehr hoher Ortsauflösung“, Photonic 6, 34-37 (2011)

[3] D. Samiec et al, Polytec GmbH, "Verteilte faseroptische Temperaturmessung und ihre Anwendungen in der chemischen Verfahrenstechnik", CITplus 3, 28-30 (2016)

[4] M. Froggatt, B. Soller, D. Gifford, M. Wolfe, "Correlation and keying of Rayleigh scatter for loss and temperature sensing in parallel optical networks", OFC Technical Digest, paper PDP 17 (2004)

[5] Figures 1-2 with kind permission from LUNA Technologies, Blacksburg, Virginia, USA 